\title{
Desafíos del Trabajo Social en un campo laboral con límites profesionales difusos
}

\author{
Sandra Iturrieta Olivares \\ Universidad Católica Silva Henríquez, Santiago de Chile
}

Desafíos del Trabajo Social en un campo laboral con límites profesionales difusos

Resumen: A partir de un estudio con enfoque cuanti-cualitativo, que consistió en la revisión de ofertas laborales para profesionales de las Ciencias Sociales publicadas durante 14 años consecutivos y sobre la base de entrevistas realizadas a trabajadores sociales en ejercicio, este artículo discute sobre el ejercicio laboral en Chile como evidencia de límites difusos entre estas profesiones, por tanto como construcción relativa. Se aborda además la pérdida de espacios laborales del Trabajo Social y su especificidad en el campo laboral. Finalmente, se insta a los trabajadores sociales a relativizar la idea de especificidad profesional para potenciar su competitividad y las posibilidades de ser considerados como trabajadores del conocimiento, más que como trabajadores genéricos.

Palabras clave: Trabajo Social. Límites difusos. Ejercicio laboral. Desafíos profesionales.

\section{Desafios do Serviço Social em um campo laboral com limites profissionais difusos}

Resumo: A partir de um estudo com enfoque quanti-qualitativo, que consistiu na revisão de ofertas laborais para profissionais das Ciências Sociais, publicadas durante 14 anos, e em entrevistas realizadas com assistentes sociais em exercício, este artigo discute o exercíco profissional no Chile, evidenciando os limites difusos entre essas profissões, como, portanto, uma construção relativa. Além disso, aborda a perda de espaços laborais do Serviço Social e de sua especifidade nesse campo. Finalmente, instiga os assistentes sociais a relativizar a ideia de especificidade profissional para potencializar sua competitividade e aumentar as possibilidades de serem considerados mais como trabalhadores do conhecimento do que como trabalhadores genéricos.

Palavras-chave: Trabalho Social. Limites difusos. Exercício laboral. Desafíos profissionais.

\section{Challenges to Social Work in a Field of Labor with Diffuse Professional Limits}

Abstract: Based on a quantitative and qualitative study that consisted in a review of the jobs available for professionals in the Social Sciences, published over 14 years, and in interviews conducted with working social workers, this article discusses the professional activity in Chile, revealing the diffuse limits among these professions, as, therefore, a relative construction. In addition, it approaches the loss of working spaces for Social Work and of its specificity in this field. Finally, it challenges social workers to consider the idea of professional specificity in a relative manner to increase their competitiveness and their opportunities to be considered more as workers who deal with knowledge than as generic workers.

Keywords: Social Work. Diffuse limits. Exercise of work. Professional challenges. 


\section{Antecedentes}

La sociedad del conocimiento y las transformaciones que está experimentando el mercado laboral han llevado a clasificar los tipos de ocupaciones, y por tanto, a los trabajadores, en diversas categorías más allá de las profesiones de origen, según el tipo de trabajo realizado y los conocimientos y habilidades que para su realización se requieren. $\mathrm{Al}$ respecto, Reich (1993) propone tres categorías: los analistas simbólicos, los trabajadores de servicios rutinarios de producción y los trabajadores de servicios a personas. De acuerdo a esta clasificación, los analistas simbólicos corresponden a "los profesionales que desarrollan trabajos de alto valor añadido, es decir, aquellos que utilizan el conocimiento como herramienta para su trabajo. Nos referimos a lo que antes conocíamos como profesionales" (INTRESS, 2007, p. 2). Mientras que los trabajadores de servicios rutinarios de producción "son los trabajadores industriales de bajo valor añadido, pero también aquellos trabajadores del sector de servicios cuyo trabajo, gracias a las nuevas tecnologías, puede desarrollarse en cualquier lugar del mundo..." (INTRESS, 2007, p. 2). Finalmente, estarían los trabajadores de servicios a personas, también denominados servicios de proximidad, que corresponden a los "trabajos del sector de servicios de bajo valor añadido, que se diferencian de los anteriores por el hecho de que el trabajador está en contacto directo con el destinatario final de su trabajo" (INTRESS, 2007, p. 2).

En la misma línea, Castells (1999) clasifica a los trabajadores como de dos tipos: los autoprogramables y los genéricos. Los primeros corresponden a los anteriormente definidos como analistas simbólicos, mientras que los genéricos corresponden a los tipificados como trabajadores de servicios rutinarios de producción y los trabajadores de servicios a personas. Según Castells, la diferencia entre ambos grupos estaría dada en que los primeros serían socialmente considerados de un modo individual, por eso serían básicamente imprescindibles cada uno de ellos. Mientras que los trabajadores genéricos serían imprescindibles como grupo, pero cada uno de ellos, indivi-

\section{En el contexto chileno, la} superposición de los límites profesionales entre las profesiones de las Ciencias Sociales se evidencia en la

tendencia del mercado laboral,

a la publicación de ofertas de trabajo bajo el rótulo de "profesional de las Ciencias Sociales", sustituyendo a aquellas dirigidas a un tipo de profesional específico. dualmente, sería considerado como prescindible por la sociedad. La razón de esta diferencia, de acuerdo a
Castells (1999), es la educación, ya que permitiría la programación hacia las tareas cambiantes del asignados a una tarea determinada no presuponen coración de información y conocimiento, más a encomendadas, por tanto, no tienen capacidad de ( simbólicos, denominados por la OIT como [...] en cualquier ámbito de actividad manipulan personas de una disciplina o una institución, 'nodos' enantes más que eruditos, nómades en la república del saber. [...] No conciben su arte sólo como la creación de conocimientos nuevos sino novedosas o de combinaciones útiles a un propósi-

En este contexto y desde una perspectiva disciplinar, la Comisión Gulbenkian para la reestructuración de las Ciencias Sociales se pregunta si "la superación la actual estructura de las disciplinas no debe ser considerada como un dilema central de las ciencias sociales en el estado actual de su evolución" (WALLERSTEIN, 2006, p. 2). A partir de ello, se ha discutido respecto de la validez de los límites disciplinares en la sociedad del conocimiento, $y$ considerando que

[...] un elemento esencial en el proceso de institucionalización de las disciplinas fue el esfuerzo de cada una de ellas por definir lo que la distinguía de las demás, especialmente, lo que la diferenciaba de cada una de las que parecían estar más próximas en cuanto al contenido en el estudio de las realidades sociales.

Es así como la comisión Gulbenkian se aboca a la reflexión respecto de las Ciencias Sociales, describiendo la evolución organizativa de éstas y dando cuenta de las distintas subdivisiones y superposiciones que comenzaron a 
producirse en ellas. Tales superposiciones se evidenciarían en los coloquios y conferencias que en las últimas décadas han tendido a reclutar a los participantes de acuerdo con un objeto de estudio concreto y sin prestar atención a la afiliación disciplinaria. Además, se basan en que un número creciente de revistas científicas de primera línea, deliberadamente, ignoran las fronteras disciplinarias. Igualmente, sustentan la hibridación de los límites profesionales en que los programas nuevos, que han surgido en el último medio siglo, suelen estar compuestos por personas tituladas en múltiples disciplinas. Finalmente, esta Comisión agrega a su argumentación que las disputas presupuestarias han potenciado que se tienda más a proponer objetos de estudio específicos que preocupaciones disciplinarias, lo que también contribuiría a la flexibilización de tales límites (WALLERSTEIN, 2006).

\section{Recuadro metodológico}

Sobre las bases anteriormente descritas se realizó una investigación empírica enmarcada en el paradigma explicativo, con un enfoque cuanticualitativo, para conocer las actuales tendencias sobre la superposición de los límites profesionales en carreras de las Ciencias Sociales desde la particular mirada del Trabajo Social.

En función de ello se revisaron los avisos de ofertas laborales publicados en el diario El Mercurio $^{1}$ de Santiago de Chile, entre los años 1997 y 2007. La revisión de estos 11 años arrojó un universo de 518 ofertas laborales para trabajadores sociales y profesionales de las Ciencias Sociales. Para evitar posibles sesgos, se complementó con otros 518 datos provenientes de avisos publicados entre los años 2008 y 2010 en la Red Virtual de empleos Cheung ${ }^{2}$. La totalidad de los datos fueron tabulados en $\mathrm{SPSS}^{3}$, sobre la base de variables categóricas, construidas a partir de los contenidos de tales avisos.

Posteriormente, se entrevistó a 56 trabajadores sociales en ejercicio de la Región Metropolitana ${ }^{4}$. Un $59 \%$ de ellos se desarrollan en organismos públicos, mientras un $41 \%$ lo hace en organismos privados. Se incluyó en este estudio a un $10 \%$ de trabajadores sociales que se desarrollan laboralmente en universidades, mientras que el resto ejerce su profesión tanto en el campo de la intervención social directa como indirecta. Se recolectaron datos hasta lograr la saturación teórica y agregando nuevas entrevistas hasta completar los porcentajes establecidos con anterioridad. La selección de informantes se hizo al azar, contactando directamente en instituciones públicas y privadas a trabajadores sociales. La validez de esta investigación estuvo dada por la saturación de los contenidos de la entrevista abierta, la que fue medida a través de un análisis categorial simple, como del mismo modo, por la triangulación de informantes que hablaron desde distintas superficies de enunciación. ${ }^{5}$

Los datos se registraron en cintas de audio que fueron transcritas íntegramente, para luego hacer una primera lectura longitudinal identificando las principales categorías presentes en los discursos. Posteriormente, en un segundo nivel de análisis, se identificaron los diferentes tópicos presentes en esas categorías. Finalmente, se estructuró una matriz de análisis considerando las categorías y subcategorías extraídas desde los discursos. Cabe mencionar que los procedimientos desplegados a lo largo de la investigación respetaron los principios éticos de la Declaración de Helsinki, contándose con el consentimiento informado de los actores sociales con quienes se desarrolló el trabajo, una vez que fueron explicitadas las finalidades y los métodos de la investigación.

\section{El ejercicio laboral como evidencia de los límites difusos entre las profesiones de las Ciencias Sociales}

En el contexto chileno, la superposición de los límites profesionales entre las profesiones de las Ciencias Sociales se evidencia en la tendencia del mercado laboral, a la publicación de ofertas de trabajo bajo el rótulo de "profesional de las Ciencias Sociales", sustituyendo a aquellas dirigidas a un tipo de profesional específico. El siguiente gráfico, figura 1, construido sobre la base de la revisión de tales ofertas, ilustra las actuales tendencias al respecto.

$\mathrm{Si}$ asumimos que el mercado laboral se comportará como hasta ahora, la tendencia lineal es la captación de "profesionales de las Ciencias Sociales", y a la disminución de las ofertas dirigidas a los trabajadores sociales, lo que evidencia los límites difusos entre tales profesiones en el mundo laboral. Además, dicha situación es posible observarse en diferentes campos laborales, donde actualmente desarrollan las mismas funciones, con iguales requerimientos profesionales, antropólogos, sociólogos, trabajadores sociales y psicólogos, entre otros tipos de profesionales de las Ciencias Sociales.

Por otra parte, a partir de los datos cualitativos es posible establecer que los límites entre las profesiones de las Ciencias Sociales, susceptibles de ser apreciados en el campo laboral, es un tema que los trabajadores sociales en ejercicio abordan a partir dos dimensiones: como una construcción relativa, por lo tanto, límites difusos; mientras que desde otra perspectiva se intenta fijar las especificidades que tendría el Trabajo Social, es decir, aquello que desde sus miradas caracteriza su ejercicio profesional. 
Figura 1 - Evolución de ofertas laborales entre los años 1997 y 2010

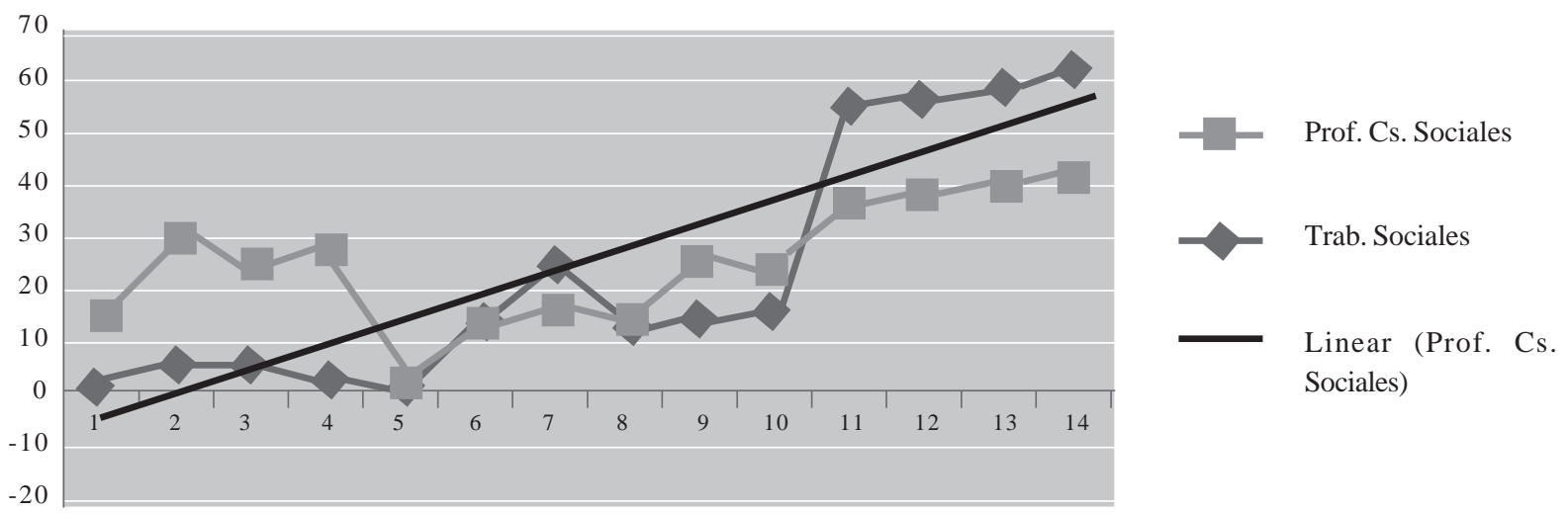

Fuente: Elaboración propia a partir de los datos recolectados desde el Diario El Mercurio de Santiago y la Bolsa virtual de la Red de empleos Cheung.

\section{Límites profesionales como construcción relativa}

El pensamiento a respecto de los límites entre las carreras de las Ciencias Sociales como construcción relativa se fundamenta, por una parte, en que el Trabajo Social en la actualidad no tendría especificidad, por eso

[...] no se puede decir esto es el Trabajo Social, esto es en lo que se mueve, acá es donde está, y eso también es positivo, es lo que pasa hoy en día con las carreras (e13).

La idea de la construcción relativa de los límites profesionales se sustenta, por una parte, en que no habría especificidad del Trabajo Social porque involucra diferentes campos disciplinares. Así,

[...] no hay especificidad desde muchos años en la carrera, lo que puede ser una ventaja y una desventaja, es una carrera muy global (e26).

Ello se debería a que

Abarcamos muchos aspectos de otras disciplinas, y eso tiene una ventaja porque somos profesionales bien completos, pero no especializarnos en algo a veces nos juega en contra (e1).

Desde otra perspectiva, se juzga positivamente la inespecificidad profesional, ya que

Hacemos de todo, y la ventaja de eso es que puedes tener una mirada global de las cosas (e26).

Desde otro enfoque, se argumenta que esta inespecificidad de las carreras profesionales, se debería a que la selección laboral se haría más bien de acuerdo a las habilidades personales, en desmedro de las especificidades profesionales, ya que

Cuando te encuentras con un trabajador social al interior de algunos equipos, ves que lo contrataron no porque necesitaban como concepto un trabajador social, sino porque se dieron cuenta de que era muy bueno en eso, entonces está más asociado a sus condiciones particulares que a su profesión (e17).

De manera que es posible encontrar trabajadores sociales

[...] en distintos niveles de intervención, ya sea más investigativo, de planificación, de proyectos, porque la gente suele entender que el trabajador social tiene que ver más con actitudes personales que con el hecho de que te determine una carrera (e17).

Desde una perspectiva similar se plantea que la inespecificidad profesional se debería a que en el ejercicio laboral, habitualmente, no existe delimitación de funciones para cada disciplina, ya que

Todos los trabajadores sociales están preparados para hacer de todo, no como otros profesionales que tienen como un área específica (e10).

\section{Así, el ejercicio profesional sería}

[...] tan amplio como problemas hay... surge un problema nuevo y siempre hay alguien que quiere resolverlo o que tiene ideas de como resolverlo (e11). 
En tal sentido, los trabajadores sociales se podrían desempeñar en distintas áreas

[...] haciendo distintas cosas, somos un poco mentholatum, ${ }^{6}$ servimos para hartas cosas, pero depende de uno (e5).

De modo que prevalece la idea de que las competencias personales priman en el ejercicio profesional, por sobre las atribuciones que se hacen a una profesión en particular.

De modo similar, el ejercicio laboral se desarrollaría mejor sobre la base de superposiciones profesionales que de especificidades disciplinares, por tanto

No hay un tema específico sobre qué profesional trabaja acá, porque vemos desde orientador familiar, trabajador social, psicólogo, sociólogo, que son las profesiones que se dan más de lo que es el caso del Programa Puente ${ }^{7}$ (e7).

De manera que esta superposición profesional se daría en diferentes campos laborales referidos a la intervención social directa, ya que

Por ejemplo, acá en el ámbito comunitário trabajan administradores públicos, profesores, trabajadores sociales, psicólogos, sociólogos... todos hacemos de todo, todos planificamos, todos asesoramos... ese es el estilo que uno aprende (e12).

Es así como

La intervención de psicólogos y trabajadores sociales es la misma... los psicólogos también hacen visitas domiciliarias, hacen intervención socioeducativa, lo mismo que hace un trabajador social (e41).

Esta superposición entre las funciones profesionales, en el ámbito de la intervención social directa, se haría evidente

Cuando buscas pega $^{8}$, ya que la misma pega que realizan los trabajadores sociales las hacen los psicólogos u otros profesionales de las Ciencias Sociales como los sociólogos, entonces el mercado se está ajustando cada vez más, no tanto a lo profesional ni a lo que tú puedas hacer bien, sino a un tema del valor que te ponga el mercado como profesional (e13).

De manera que los límites profesionales difusos, entre las profesiones de las Ciencias Sociales, se manifestarían en el ejercicio profesional del ámbito de la intervención social directa, en que
Estamos como muy al límite de muchas profesiones en las Ciencias Sociales, la psicología, la sociología [...] es un hilo superfino que nos separa porque estamos insertos en muchos ámbitos comunes, entonces, es como imposible en la actualidad tener un área específica, estamos en muchas áreas, pero esas áreas también limitan mucho con otras profesiones de las Ciencias Sociales (e25).

\section{Pérdida de espacios laborales del Trabajo Social en el contexto de límites profesionales difusos}

Desde un prisma diferente, se plantea que los límites difusos entre el Trabajo Social y las otras profesiones de las Ciencias Sociales se debería a que el primero ha perdido espacios laborales. Esta pérdida de plazas laborales sería consecuencia, por um lado, de características internas del Trabajo Social, y por otro lado, de la expansión de otras carreras de las Ciencias Sociales.

De manera que lo que estaría influyendo en la pérdida de espacios laborales sería, por una parte, que

En la formulación y la evaluación de proyectos, o sea, en el proceso continuo de un proyecto que tiene que ver con una demanda potente del mercado laboral, hay pocos profesionales que saben sobre eso, el Trabajo Social ha perdido espacio en ese sentido, también ha perdido espacio trabajando con la gente, teniendo acceso directo a las comunidades o a los grupos vulnerables, a veces, se ocupan en este ámbito a otros profesionales como sociólogos e incluso antropólogos, cuando nosotros deberíamos manejar esto al dedillo y generar referencias para los demás profesionales (e26).

Esta falta de preparación profesional en algunas áreas de desempeño laboral provocaría que

[...] haya otras profesiones que han pasado sobre nosotros, eso también nos juega en contra, porque el área de psicología, por ejemplo, tiene sus propias teorías y ha invadido también nuestro terreno, ahora el campo laboral para los psicólogos se amplió y de hecho hay cosas que los psicólogos hacen y que son del área del trabajador social, entonces falta ser más específicos, la carrera está muy amplia y siempre enfocada a lo mismo, a la ayuda [...] sería bueno generar conocimientos desde el área social (e32).

Otro factor influyente sería la escasa producción de conocimientos desde el Trabajo Social, lo que se traduciría en que 
Se han perdido espacios laborales porque nos hemos quedado un poco pasivos en el tema de la investigación, en los temas de la sistematización de la práctica social, por lo tanto, de apropiarse de ciertas prácticas, y otras disciplinas han tomado nuestra práctica y la han sistematizado, han hecho investigaciones e incluso nos capacitan en esos temas $[. .$.$] los espacios que hemos perdido han sido$ por falta de rigurosidad en las acciones, en el trabajo profesional, y también, porque siempre tendemos a disminuirnos frente a otros profesionales (e45).

De modo que la escasa producción de conocimientos, sumada a la sensación de menoscabo profesional y a la vaguedad en el ejercicio profesional, explicarían la pérdida de plazas laborales. Al mismo tiempo, estas insuficiencias se derivarían de

La proliferación de la formación académica, que actualmente es muy comercial, y por lo tanto, se ha desmejorado el profesional que sale hoy en día al mercado; eso ha permitido que otras profesiones, al no hacerlo tan bien nosotros en el terreno, nos aborden, y hemos perdido espacios laborales [...] por ejemplo, los concursos públicos ahora ya no vienen dirigidos a las profesiones, sino que a un cargo [...] hoy en día en el ámbito social no hay límites (e40).

Es así como los campos profesionales, en la actualidad, tendrían límites difusos. En ese contexto,

El Trabajo Social se ve como una profesión muy amplia que tenía que ver con todo y comenzaron a aparecer ciertas profesiones que empezaron a indagar en nuestro propio ámbito de trabajo (e21).

\section{Todo ello provocaría que}

La especificidad del Trabajo Social se vaya perdiendo, incluso en el campo laboral ya se habla de: necesito un profesional de las Ciencias Sociales, ni siquiera se está especificando un trabajador social, un sociólogo, un psicólogo, piden que tenga estudios en Ciencias Sociales, entonces el tema de la especificidad es complejo (e24).

\section{Especificidad del Trabajo Social en el contexto laboral}

Los entrevistados conciben la especificidad del Trabajo Social sustentada en premisas tales como: una mirada profesional distintiva; un perfil característico; la particularidad en el trato directo con las personas; la existencia de funciones definidas para este tipo de profesionales; y una inespecificidad que sería, a su vez, su especificidad. Conjuntamente, la especificidad profesional estaría dada por el trabajo en el terreno; la aplicación de determinados modelos de intervención; conocimientos sobre familias; la elaboración de informes sociales y la asistencia social.

Respecto del enfoque del Trabajo Social, los profesionales entrevistados consideran que lo distintivo de los trabajadores sociales sería

[...] una mirada global, eso es lo que podemos $\operatorname{aportar}(\mathrm{e} 26)$.

Ello implicaría el análisis

[...] integral del origen y causas de los problemas, tanto desde la objetividad como desde la subjetividad, eso es lo que nos distingue de las carreras afines (e47).

Asimismo, dicha mirada global se expresaría en un

[...] enfoque de la realidad que no lo tienen las otras profesiones, porque vamos desde lo micro a lo macro (e42).

De modo que los entrevistados juzgan que su profesión tendría

Una especificidad en la mirada, por eso el Trabajo Social aporta en ampliar las perspectivas, en mostrar a otros profesionales que hay otras formas, en dar una mirada más integral y globalizante de los problemas, de las situaciones, de las personas (e45).

Paralelamente, la particularidad del Trabajo Social estaría en que el mismo desarrollaría

[...] una mirada distinta a otras profesiones, más que nada por un tema de cómo intervenir en una cierta situación (e8).

Es así como

La especificidad del Trabajo Social es justamente que el trabajo con las personas le permite entender que hay múltiples variables que afectan su actuar (e3). [...] puede ser subjetivo, por ejemplo, cuando hay un infractor de la ley es muy distinta la propuesta de un trabajador social, la de un sociólogo y la de un psicólogo que lo ven solamente desde el punto de vista individual, en cambio, el trabajador social sabe la relevancia del entorno, lo sociocomunitario, las familias, todo ese tipo de cosas [...] esa es la gran diferencia (e3).

Sobre esas bases, los trabajadores sociales entrevistados consideran que 
Podemos hacer una intervención superbuena con la gente, pero uno está dispuesto a ayudar, no así un sociólogo que puede hacer una investigación y eso queda ahí (e8).

\section{Ello se debería a}

[...] que los sociólogos analizan más la situación, mientras que nosotros la analizamos, pero la tratamos de resolver, no nos quedamos ahí (e37).

Otro de los elementos realzados por los trabajadores sociales entrevistados corresponde al perfil de quienes ejercen la profesión, que se caracterizarían por

[...] un compromiso ético, personal, de buen corazón también, eso se ve cuando se está en intervenciones con otros profesionales, porque aunque ellos quieran dar su pincelada desde el punto de vista social, no tienen esa sensibilidad que por formación uno tiene... hay un matiz que pone el trabajador social a todos los temas y que ellos no lo ponen (e49).

Dicho matiz estaría dado porque este tipo de profesionales tendría

[...] ciertos lineamientos bien claros, pero más desde lo ideológico que desde lo social y eso es super potente y marcador como profesionales en general, es como que nos identifica, lo ideológico que nos marca a la mayoría de los que desempeñamos esta carrera, en cualquier rubro y hasta en una empresa... lo tenemos marcado de forma diferente a un antropólogo o un sociólogo, porque ellos igual pueden seguir ciertas líneas, pero la nuestra siempre va más asociada a un sentido social (e13).

En concordancia con lo anterior, se argumenta que la especificidad del Trabajo Social estaría en el perfil profesional, en el sentido de que

Nuestras fortalezas están en la empatía con la gente (e12).

\section{$\mathrm{Y}$ en ser}

[...] más cordiales, en la atención de escucha, eso nos diferencia de las otras profesiones que son más frías, más cortantes (e9).

Paralelamente, se estima la intervención social directa como una particularidad del Trabajo Social, puesto que considera que

Como disciplina, lo que nos particulariza en comparación con la psicología y la sociologia es el trato directo con las personas en todos los ámbitos, porque vemos todos los ámbitos de la vida de una persona (e47).

Por tal razón, se considera que

Los trabajadores sociales deberíamos ser actores privilegiados en la aplicación de programas que impliquen el trato directo con la gente, yo creo que en eso nosotros tenemos experiencia (e14).

En consecuencia, la especificidad del Trabajo Social estaría dada por su perfil práctico y de trabajo en el terreno. En tal sentido, se plantea que

Lo que nos diferencia de cualquier otra carrera son los conocimientos en la práctica, hoy en día el de la vulnerabilidad social, nosotros conocemos realmente el trabajo con la gente que se encuentra en situaciones críticas (e46).

\section{Así, la diferencia con el}

[...] resto de los profesionales es que ellos están en sus escritorios y nosotros somos del terreno (e33).

Desde otro punto de vista, pero siempre en el tema de la intervención social directa, la especificidad del Trabajo Social estaría dada en la aplicación de determinados modelos. De manera que si

[...] vamos a hacer una intervención utilizando el modelo ecológico, en un determinado sector de la comuna, el que podría hacer eso es un trabajador $\operatorname{social}(\mathrm{e} 12)$.

Asimismo, la especificidad en la intervención social directa estaría en la intervención en crisis puesto que

Somos el primer contacto para la gente, entonces la persona cuando tiene un problema al primero que recurre es al trabajador social, nosotros recibimos todo lo que la gente nos dice y ahí lo vamos derivando donde tengamos que mandarlo (e44).

Igualmente, la mediación sería una particularidad de la profesión

[...] porque como trabajadora social tengo que tener las habilidades para mediar en un conflicto social (e6).

En el mismo sentido, se plantea que

La visita domiciliaria es una herramienta que debería ser privilegiada por el Trabajo Social y para el Trabajo Social (e14). 
Otro de los componentes de la especificidad del Trabajo Social serían las funciones atribuidas a este tipo de profesionales, ya que

La formulación de diagnósticos o evaluaciones, no se las piden a ningún otro profesional más que al trabajador social, que debe hacer las pautas de trabajo que otros profesionales aplican para hacer diagnósticos, hacer la evaluación de los proyectos, los seguimientos (e50).

Entre tanto, otra de las características particulares de esta profesión correspondería a sus labores en el ámbito familiar, ya que en dicho terreno

No hay profesional que sepa más que el trabajador social en temas de dinámicas familiares, en cómo intervenir con familias (e18).

De modo que el Trabajo Social tendría

La capacidad como profesión de poder intervenir en las familias, en lo que son adentro, manejando el tema del sistema, los subsistemas, nosotros vemos el subsistema pequeñito de la sociedad que es la familia (e39).

\section{Al mismo tiempo, habría otros ámbitos}

[...] que son superespecíficos para el Trabajo Social, como la asistencia social (e6).

\section{Además del}

[...] tema de los beneficios sociales, de la activación de redes, los informes socioeconómicos... pero tenemos pocas cosas que son propias (e16).

En cualquier caso,

Hay un campo, que es el asistencial, que aún no ha sido colonizado por otra profesión, que es como el único nicho que tenemos hoy en día [...] la gente entiende que ese es un campo de desarrollo de un trabajador social, único y exclusivo de un trabajador social (e17). Lo que es propio, en el trabajador social, es justamente el tema de la asistencia social (e43).

Ello se plasmaría además

En un Estado subsidiario, que está lleno de políticas sociales [...] para que las personas puedan acceder a subsidios, a programas especiales, ahí se ha especificado mucho la labor del trabajo social (e36).

En tal sentido, la especificidad estaría en la elaboración de informes sociales y que sería
Un campo propio, porque el trabajo que hacemos nosotros no lo hace nadie más, porque yo no he conocido otro profesional que haga visitas domiciliarias, que haga un informe social y que lo validen (e33).

\section{Ello se debería a que}

Un informe social solamente lo sabe hacer un trabajador social, eso es lo más específico, otros no pueden firmar porque no son profesionales que estén preparados para eso (e28).

Desde una perspectiva totalmente diferente, se plantea que la inespecificidad sería la especificidad de la profesión ya que los trabajadores sociales

Sabemos de todo un poquito, pues sabemos enfrentar cualquier circunstancia del trabajo que se lleve a cabo, estamos capacitados en cuanto a conexiones, porque a uno le hacen un perfil en la universidad de que tienes que ser buen lector de las cosas sociales, entonces uno tiene siempre un conocimiento, te enfrentas a un trabajo y dices, pero esto yo lo podría ver por este lado o por este otro lado, uno tiene las herramientas para ir investigando y como somos medio mentholatum, podemos hacer esas conexiones (e19).

Por tanto, sería

[...] bueno que sepamos un poco de todo y que abordemos la problemática social desde una perspectiva mucho más interdisciplinaria que la labor de los abogados, del psicólogo o del sociólogo; tenemos la capacidad por nuestra inespecificidad de ver la problemática humana de una manera distinta (e4).

\section{Conclusiones}

En los discursos de los trabajadores sociales entrevistados aparecen claramente demarcadas dos (2) posturas referidas a la superposición de límites profesionales en el contexto laboral: por una parte, se defiende la idea de la especificidad del Trabajo Social, y por otro lado, tales límites se ven como construcciones relativas que pierden validez en el ejercicio laboral, manteniéndose solo como delimitaciones disciplinarias impuestas institucionalmente. Ello es coherente con la denominación de los trabajadores conforme al tipo de trabajo realizado y a las habilidades que requieren para desarrollar una determinada función, más que la denominación por la profesión de origen. Quienes convergen en esta perspectiva identifican el ámbito de la intervención microsocial como el espacio en que más 
se evidencia la superposición profesional, ello explicaría que el mercado laboral, actualmente, requiere más que conocimientos, competencias personales y profesionales, es decir, se necesitaría más bien un "trabajador del conocimiento" que un profesional específico.

Desde la mirada de la especificidad del Trabajo Social, los requerimientos comunes a las profesiones de las Ciencias Sociales potenciaría la competencia por plazas laborales y llevando a los profesionales a la especialización temática, siendo considerada la inespecificidad como un factor que dificulta el acceso a un puesto de trabajo. Es así como se representa problemático el desdibujamiento de los límites profesionales, ya que se perderían plazas laborales como producto de ello. Tal pérdida es explicada por la expansión de otras carreras de las Ciencias Sociales, como por factores internos a la profesión referidos a: la escasa producción de conocimientos desde el Trabajo Social; la sensación de menoscabo profesional; la proliferación del mercado de la formación de trabajadores sociales, y la falta de preparación académica en algunas áreas del desempeño laboral, lo que llevaría a la vaguedad en el ejercicio profesional.

Desde otro punto de vista, los trabajadores sociales entrevistados sustentan su especificidad en una mirada global de las realidades a intervenir; en su perfil práctico; en las habilidades de trato directo, y siempre desde la relación de ayuda basada en componentes éticos e ideológicos con marcados énfasis asistencialistas. Todo ello es coherente con las demandas del actual mercado laboral, ya que las competencias más demandadas a estos profesionales corresponden a las relativas al buen convivir y relacionarse, seguidas de acción y gestión, por lo que la mirada crítica tan frecuentemente aludida en el discurso profesional queda reducida a una intencionalidad no plasmada en el quehacer profesional. Sin embargo, todas las características mencionadas por estos trabajadores sociales son compartidas por otras profesiones de las Ciencias Sociales, tales como la sociología en su vertiente profesionalizante, por lo tanto, este sería un discurso que habría que comenzar a relativizar.

Por otro lado, efectivamente, en los últimos años se ha producido una proliferación de escuelas de trabajo social en Chile, no existiendo aún evidencias cuantitativas de saturación de esta profesión en el mercado laboral, como tampoco existen evidencias empíricas de la pérdida de plazas laborales para trabajadores sociales, ya que según cifras oficiales publicadas en el Ministerio de Educación chileno, esta es la profesión, entre las que confluyen en la intervención social directa e indirecta, con la tasa más alta de empleabilidad, pero con los menores salarios ${ }^{9}$.

Los factores mencionados son influyentes en el Trabajo Social chileno en la actualidad, pero que no explican por sí solos la superposición profesional en el campo de la intervención social directa, siendo evidente la despolitización de las concepciones profesionales en los discursos analizados, ya que no se mencionan variables estructurales que permitan explicar la superposición de los límites profesionales en el ejercicio laboral, en un contexto tanto de precarización de las condiciones laborales para las profesiones de las Ciencias Sociales en general, como del aumento de los niveles de cesantía juvenil y de una concepción fragmentaria de las políticas sociales, en un país neoliberal como Chile.

Finalmente, sobre los antecedentes recabados en esta investigación, es posible sostener que el Trabajo Social chileno debe enfrentar los desafíos que la sociedad del conocimiento le plantea, referidos a situarse en el escenario laboral actual que evidencia los límites profesionales difusos, para ampliar la mirada más allá de las fronteras profesional/disciplinar autoimpuestas. Toda vez que ello ocurra, será posible competir con otros profesionales como trabajadores del conocimiento más que como expertos técnicos neutrales, ya que actualmente los intentos por salvaguardar lo considerado propio son infructuosos, limitando más bien las posibilidades de actuación profesional y del desarrollo profesionaldisciplinar. Igualmente, el Trabajo Social chileno estaría exhortado a lograr una vigilancia epistemológica en relación a la metodología empleada en la producción de conocimientos, a resguardar la coherencia entre las teorías empleadas para el análisis de los fenómenos sociales y el concepto de ciencia que las sustenta. Asimismo, para ampliar su mirada sobre la producción de conocimientos aún constreñida por una perspectiva eficientista, vinculada a la intervención microsocial que continúa dificultando su reconocimiento social como profesión y como disciplina. Todo ello dotaría de realidad a la mirada crítica de la profesión, potenciando su competitividad frente a otras profesiones de las Ciencias Sociales, pudiendo ser valorados como trabajadores del conocimiento más que como trabajadores genéricos.

\section{Referencias}

BRUNNER, J. Educación superior en América Latina: Cambios y desafíos. Chile: Fondo de Cultura Económica, 1990.

CASTELLS, M. La era de la información. Chile: Fondo de Cultura Económica, 1999.

INTRESS-Instituto de Trabajo Social y Servicios Sociales. España.

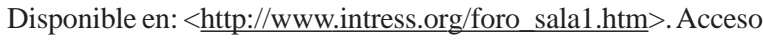
en: 16 sept. 2009. 
REICH, R. El trabajo de las naciones. Hacia el capitalismo del siglo XXI. Traducción de Federico Villegas. Buenos Aires: Vergara, 1993.

WALLERSTEIN, I. Abrir las Ciencias Sociales. Informe de la Comisión Gulbenkian para la reestructuración de las Ciencias Sociales. México: Siglo XXI, 2006.

\section{Notas}

1 El diaro El Mercurio de Santiago se encuentra disponible en la Hemeroteca de la Biblioteca Nacional de Santigo de Chile.

2 Para más detalles sobre la Red Virtual de Empleos Cheung ver: $<$ http://lists.comunitarios.cl/listinfo.cgi/bolsade trabajocheungcomunitarios.cl>. Acceso en: 15 sept. 2010.

3 El SPSS es un software para la elaboración de análisis estadísticos de matrices de datos. A través de su uso es posible generar tablas, gráficos y todas las demás herramientas necesarias para la realización de un análisis descriptivo e inferencial a partir de correlaciones.

4 La opción por la Región Metropolitana de Chile se debió a criterios de accesibilidad sustentados, además, en la mayor concentración de ofertas tanto para la formación profesional universitaria como de puestos laborales para este tipo de profesionales en esta región.

5 Con vistas a asegurar el anonimato de los informantes, las entrevistas fueron numeradas al azar en forma correlativa del 1 al 56. Cada párrafo textual extraído desde las entrevistas fue ideintificado con la letra e, seguida del número correspondiente a ésta.

6 El ungüento Mentholatum, particularmente popular en países como el Perú y Chile, ha derivado en una palabra para denominar a las personas que realizan múltiples funciones. Disponible en: <http://es.wikipedia.org/wiki/Mentholatum>. Acceso en: 12 mayo 2010.

7 El Programa Puente depende del Ministerio de Planificación y Cooperación y está destinado a familias que viven en condiciones de extrema pobreza. Para más detalles ver: <http:// public.programapuente.cl $>$. Acceso en: 12 mayo 2010.

8 Chilenismoque significa trabajo.

9 Para más detalles ver: <http://www. futurolaboral.cl/ index.php?option $=$ com content $\&$ view $=$ article $\&$ id $=$ 46\&carrID=181\&Itemid=29>. Acceso en: 12 mayo 2010.

\section{Sandra Iturrieta Olivares}

siturrie@ucsh.cl

Trabajadora Social

Doctorante en el Análisis de los Problemas Sociales de las Sociedades Avanzadas, Escuela de Trabajo Social de la Universidad Católica Silva Henríquez, Santiago de Chile

\author{
Universidad Católica Silva Henríquez \\ Escuela de Trabajo Social \\ General Jofré 462 \\ Santiago de Chile
}

